\title{
Overcoming qRT-PCR interference by select carbon nanotubes in assessments of gene expression
}

Sara T. Humes ${ }^{1, *}$, Shannon Hentschel2,*, Candice M. Lavelle', L. Cody Smith ${ }^{3}$, John A. Lednicky ${ }^{1}$, Navid B. Saleh ${ }^{4}$, and Tara Sabo-Attwood ${ }^{1}$ ${ }^{1}$ Department of Environmental and Global Health, University of Florida, ${ }^{2}$ Department of Molecular Genetics and Microbiology, University of Florida, ${ }^{3}$ Department of Physiological Sciences, University of Florida, and ${ }^{4}$ Department of Civil, Architectural, and Environmental Engineering, University of Texas at Austin

${ }^{*}$ S.T.H. and S.H. contributed equally to this work.

BioTechniques 63:81-84 (August 2017) doi 10.2144/000114578

Keywords: qRT-PCR interference; carbon nanotubes; gene expression

Supplementary material for this article is available at www.BioTechniques.com/article/114578.

Nanomaterials (NMs) of various types, including carbon nanotubes (CNTs), can interfere with standard quantitative real-time PCR (qRT-PCR) assays, resulting in inaccurate gene expression measurements; however, the precise step in the GRT-PCR pipeline where this interference occurs has not been well described. Here, we investigated where in the process surface-oxidized multi-walled CNTs (oxMWNTs) inhibited qRT-PCR measurement of the expression of the housekeeping gene GAPDH and explored several strategies to minimize such inhibition. We determined that the interference occurred during the reverse transcription (RT) step and found that doubling reaction reagents or adding BSA successfully mitigated the inhibition. We observed assay interference in the presence of CNTs that were surface-oxidized, but pristine CNTs did not cause the same level of interference. These results highlight the importance of monitoring qRT-PCR assays for interference by CNTs that differ by surface chemistry, as these NMs are commonly used in gene expression assays at concentrations that we have shown to be inhibitory.

The increased use of nanomaterials (NMs) in consumer and industrial products has prompted assessments of their effects on biological systems. A common endpoint for examining cellular responses to NMs is gene expression, which is frequently measured by quantitative real-time PCR (qRT-PCR). Several types of NMs have been reported to alter the efficiency and specificity of PCR (1-11); however, dependent on dose, size, functionalization, and surface area, this alteration has been hypothesized to occur through fluorescence quenching and adsorbing or inactivating reagents (2,3,12-14). For example, polyethyleneimine (PEI)-modified carbon nanotubes (CNTs) have been used to increase PCR efficiency by enhancing electrostatic interactions between CNTs and PCR components (1); however, Sang et al., (13) reported that several types of CNTs inhibit PCR through fluorescent dye quenching, and Cui et al., (3) noted that CNTs at concentrations greater than $3.0 \mu \mathrm{g} / \mu \mathrm{l}$ inhibited PCR through sorption of assay components. Though there is evidence of both beneficial and detrimental effects of NMs on qRT-PCR, defining which type of CNT, the step of qRT-PCR most impacted, and recommendations to overcome undesirable effects have not been well described.

Here, we report that inhibition of qRT-PCR by surface-oxidized multiwalled CNTs (oxMWNTs) occurs during the reverse transcription (RT) step of the qRT-PCR process. This was first observed in studies where a dose-dependent repression of GAPDH mRNA expression occurred in small airway epithelial cells (SAECs) exposed to oxMWNTs. Repression occurred at a dose of $20 \mu \mathrm{g} / \mathrm{mL}$, which is in line with, or lower than, commonly reported doses. This did not occur with other types of CNTs such as pristine SWNTs (Southwest Nanotechnologies, Inc., Norman, OK) (Figure 1B) or carbon black (data not shown). These data led to investigations aimed at defining the stage in the GRT-PCR pipeline where inhibition occurred. Performing qRT-PCR requires several steps that start with good quality RNA followed by RT to synthesize cDNA and then gene-specific PCR amplification.

Our gene expression assessments were performed on RNA isolated using a phenol:chloroform extraction method and not a column-based kit. Using this method affords us greater RNA yields, particularly when using NM-exposed cells and tissues. Assured that we were starting with high-quality RNA (as determined by 260/280 absorbance ratio $>1.9$ and RNA integrity number > 9.0) (Supplementary Figures S1A and S1B), cDNA was synthesized from 250 ng RNA (qScript cDNA Synthesis Kit; Quantabio, Beverly, $M A$ ) isolated from SAECs that had been treated with: (i) control (vehicle pluronic), (ii) $50 \mu \mathrm{g} / \mathrm{mL}$ pristine SWNTs, and (iii) $20 \mu \mathrm{g} /$

\section{METHOD SUMMARY}

Interference of quantitative real-time PCR (qRT-PCR) by surface-functionalized carbon nanotubes (CNTs) can be mitigated by decreasing the concentration of RNA in combination with either doubling reaction reagents or adding BSA to the reverse transcription (RT) step. 
$\mathrm{mL}$ oxMWNTs. The cDNA was utilized in a variety of experiments to assess efficiency and dye quenching in the qRT-PCR step.

Using 10-fold serial dilutions of cDNA, we constructed standard curves based on GAPDH mRNA expression, which showed reduced amplification in the oxMWNTexposed samples (Figure 1C) compared with controls (Figure 1A) and SWNT-exposed (Figure 1B) samples. Regression analysis of RNA starting concentration and $\mathrm{C}_{\mathrm{q}}$ values for control and oxMWNT-treated groups produced slopes that were nearly parallel, suggesting qRT-PCR primer efficiency was not greatly impacted (Figure 1D). To rule out dye quenching by the oxMWNTs, amplicons were visualized on an ethidium bromidestained gel. No bands were observed in oxMWNT-exposed samples after 25 and 30 cycles, while amplicons were visible in control samples (Figure 1E). PCR products were detected after 40 cycles in control and oxMWNT-exposed samples, but to a lesser extent for the latter (Figure 1E). These data support the theory that dye quenching was not likely responsible for the reduced expression measurements and suggest that interference with the RT step, resulting in decreased amounts of cDNA available for the qRT-PCR reaction, was a plausible explanation.

To verify this hypothesis, we constructed an RT inhibition curve by reverse transcribing a range of RNA concentrations (213.6 pg-3500 ng) and performing qRT-PCR to measure GAPDH expression. We observed reduced amplification in oxMWNT-exposed samples when $>218.75$ ng of template RNA was used (Figure 1F). Therefore, decreasing the amount of RNA template used for the RT reaction can reduce the inhibition observed, likely due to dilution of CNTs.

As CNTs are considered highly sorptive, we took several approaches to address possible reagent sequestering in the RT step. First, the RT reaction components were doubled while keeping the amount of RNA template consistent (400 ng) for each reaction. This resulted in less inhibition, as shown by a decreased $\mathrm{C}_{\mathrm{q}}$ for oxMWNT-exposed samples in the doublecompared with the single-component reaction; however, there was still a significant difference compared with controls for both reaction conditions (Figure $2 \mathrm{~A}$ ). Doubling the reaction did not have any effect on SWNTs or the low-dose exposure to oxMWNTs (Figure 2A). We then lowered
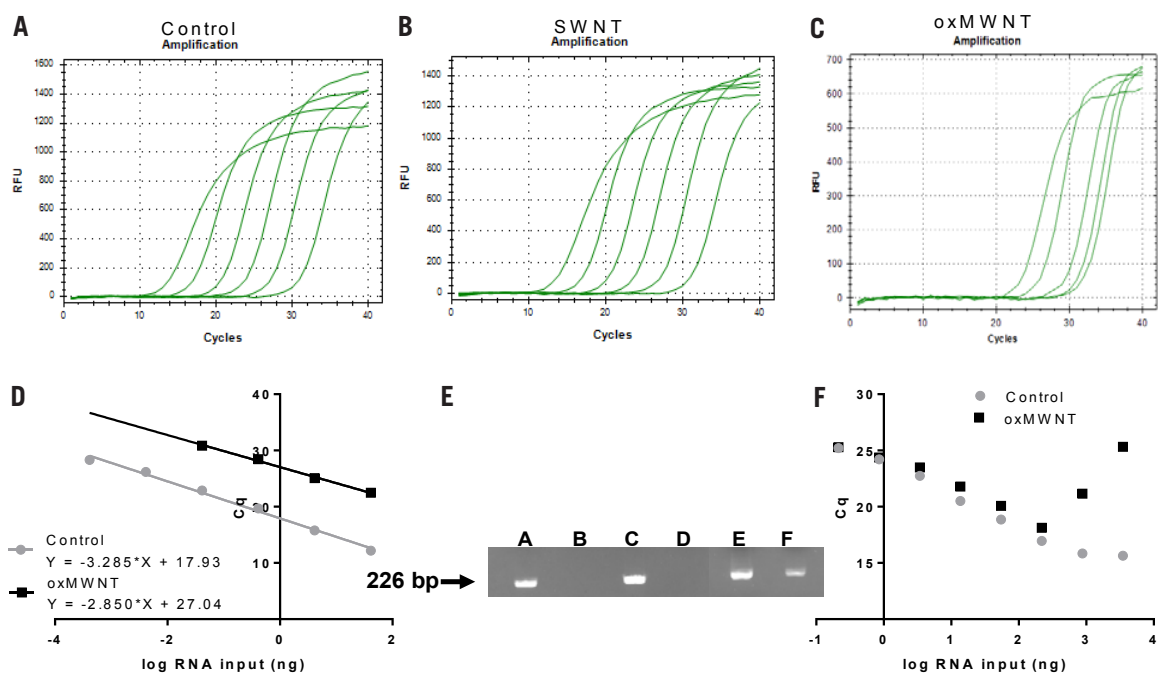

Figure 1. Inhibition of qRT-PCR GAPDH gene expression measurements for oxidized multi-walled carbon nanotube (oxMWNT)-exposed samples. Representative amplification curves for a qRT-PCR standard curve (10-fold dilution) for GAPDH expression for (A) control vehicle-, (B) $50 \mathrm{\mu g} / \mathrm{mL}$ pristine single-walled carbon nanotube (SWNT)-, and (C) $20 \mu \mathrm{g} / \mathrm{mL}$ oxMWNT-exposed cells using cDNA synthesized from $250 \mathrm{ng}$ of RNA by reverse transcription (RT). Only five curves are presented in (C) because expression was not detectable in the other dilutions, likely due to low concentrations of starting material and the observed inhibition. (D) Regression analysis of qRT-PCR standard curves in (A) and (C). The lowest dilutions were not plotted due to undetectable levels of expression. (E) Visualization on an ethidium bromide-stained agarose gel of GAPDH amplicons from control samples (lanes A, C, and E) and $20 \mu \mathrm{g} / \mathrm{mL}$ oxMWNT-exposed samples (lanes B, D, and F). Lanes A and B show products after 25 cycles, lanes $C$ and D show products after 30 cycles, and lanes $E$ and $F$ show products after 40 cycles. The GAPDH PCR product was 226 bp. (F) RT inhibition curve for GAPDH expression in 20 $\mu \mathrm{g} / \mathrm{mL}$ oxMWNT-exposed samples $(n=3)$ compared with the RT inhibition curve for control samples $(n=3)$.
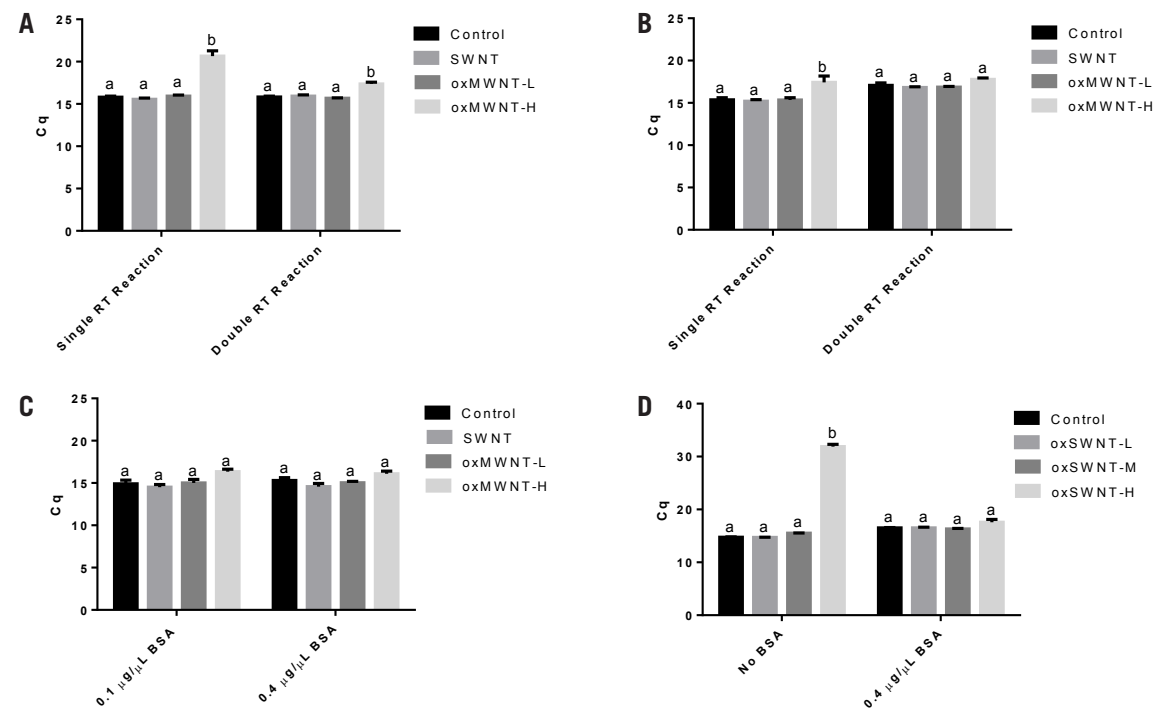

Figure 2. Results of different strategies for reducing reverse transcription (RT) inhibition. Small airway epithelial cells (SAECS) were exposed to various concentrations of oxidized multi-walled carbon nanotubes (oxMWNTs), represented as low- $(\mathrm{L}, 2 \mu \mathrm{g} / \mathrm{mL})$ or high- $(\mathrm{H}, 20 \mu \mathrm{g} / \mathrm{mL})$ dose, and single-walled carbon nanotube (SWNTs) $(50 \mu \mathrm{g} / \mathrm{mL})$. (A) Average $\mathrm{C}_{q}$ for each treatment group using $400 \mathrm{ng}$ of RNA template in singleand double-volume RT reactions. The $\mathrm{C}^{\mathrm{a}}$ for the high-dose MWNT-exposed sample is significantly different from the control for the single-volume RT reaction $(P \leq 0.0001)$ and the double-volume RT reaction $(P \leq$ 0.001 ). (B) Average $C$ for each treatment group when using $250 \mathrm{ng}$ of RNA template in single- and doublevolume RT reactions. The high-dose oxMWNT-exposed sample is significantly different from the control for the single-volume RT reaction $(P \leq 0.05)$ but not for the double-volume RT reaction. (C) Average $\mathrm{C}$ for each treatment group when adding BSA to a final concentration of $0.1 \mu \mathrm{g} / \mu \mathrm{l}$ or $0.4 \mu \mathrm{g} / \mu \mathrm{l}$ in the RT reaction. The differences among the means of the treatment groups for each BSA dose are statistically significant using a one-way ANOVA $(P \leq 0.05)$ but not when using post-hoc tests. (D) Average $C$ for control, $0.2 \mu \mathrm{g} / \mathrm{mL}$ oxidized SWNT (oxSWNT-L), $2 \mu \mathrm{g} / \mathrm{mL}$ oxSWNT (oxSWNT-M), and $20 \mu \mathrm{g} / \mathrm{mL}$ oxSWNT (oxSWNT-H) samples in a typical RT reaction compared with a RT reaction containing $0.4 \mu \mathrm{g} / \mu \mathrm{l} \mathrm{BSA}$. For the typical RT reactions, the $20 \mu \mathrm{g} / \mathrm{mL}$ oxSWNT C is significantly different from the control. All treatment groups include three samples, and the error bars represent SE. Statistical differences were determined using a one-way ANOVA with Dunnett's multiple comparisons test $(P \leq 0.05)$, with statistically significant groups denoted with different letters. 
the concentration of the starting RNA. When using 250 ng of template RNA and doubling the reaction components, the inhibition observed in the high-dose oxMWNTexposed samples was eliminated (Figure 2B). These experiments demonstrated that both reducing the amount of RNA template and doubling the $\mathrm{RT}$ reaction components reversed the repression of gene expression measurement by qRT-PCR, an observation likely due to reduced CNT carryover, the sorptive nature of CNTs, and sequestration of reaction components.

While successful, doubling $\mathrm{RT}$ reaction components is cost-prohibitive. Therefore, we explored the use of BSA, as previous reports have shown that it can reduce interference caused by gold nanoparticles in standard PCR $(14,15)$; however, no studies to our knowledge have demonstrated its usefulness in the RT step. To test this, we employed BSA $(0.1 \mu \mathrm{g} / \mu \mathrm{l}$ and $0.4 \mu \mathrm{g} /$ $\mu l)$ in the RT reaction for control, SWNT-, and oxMWNT-exposed samples. The addition of BSA reduced $\mathrm{C}_{q}$ values (Figure 2C) and decreased the $\log _{2}$-transformed average cycle difference between oxMWNT samples and control samples from 5.34 with no BSA to 2.81 in the presence of 0.1 $\mu \mathrm{g} / \mu \mathrm{l} \mathrm{BSA}$ and 1.80 in the presence of 0.4 $\mu \mathrm{g} / \mu \mathrm{l}$ BSA (Supplementary Figure S2). The effectiveness of each scenario (single RT, double RT, $0.1 \mu \mathrm{g} / \mu \mathrm{l}$ BSA, and $0.4 \mu \mathrm{g} / \mu \mathrm{l}$ BSA) was evidenced by the $\log _{2}$-transformed average cycle difference between CNT-exposed samples and control samples for both the oxMWNT-exposed and SWNTexposed groups (Supplementary Figure S2). Furthermore, the addition of BSA corrected the normalization of a selected gene target (IFIT3) and in fact changed the overall pattern of the expression data (Supplementary Figure S3).

As noted above, inhibition of qRT-PCR during GAPDH amplification only occurred in samples exposed to surface-functionalized (oxidized) MWNTs and not pristine SWNTs. This suggested that nanoparticle properties such as surface chemistry and structural composition (single- versus double-walled) may contribute to the qRT-PCR inhibition. To answer this question, we performed experiments with oxidized SWNTs (oxSWNTs) and observed inhibition of qRT-PCR that was similar to that caused by oxMWNTs. Furthermore, inhibition by oxSWNTs was rescued by the addition of BSA (Figure 2D). These data imply that the inhibition in the RT step likely involves surface oxidation, since no inhibition was observed with pristine SWNTs. Although there are differences in properties, such as aggregate size under biological conditions for the CNT types (SWNTs: $120 \mathrm{~nm}$; MWNTs: $200 \mathrm{~nm})$, their similar percent surface oxidation ( 10\%) suggests this to be a driving property of their sorptive capacity. Acid-functionalized (or oxidized) CNTs have been shown to bind proteins much more strongly compared with pristine CNTs, as the positively charged surface moieties on proteins show stronger electrostatic interactions with functionalized nanotubes with negatively charged oxide groups $(16,17)$.

Here, we demonstrated that the addition of BSA to the RT step is a cost-effective strategy to reduce inhibition caused by surface oxidized CNTs in qRT-PCR. Such observations are significant as NMs are commonly used at concentrations that we have shown to be inhibitory to qRT-PCR assays. It is especially prudent to note that not all CNTs equally inhibit qRT-PCR, and variations in particle characteristics, such as surface chemistry, need to be carefully monitored for assay interference in order to ensure the validity and accuracy of gene expression data.

\section{Author contributions}

T.S.A., C.M.L., and J.A.L. conceived the idea for the study. S.T.H., L.C.S., and S.H. performed the experiments. S.T.H., C.M.L., S.H., and T.S.A. analyzed data and wrote the manuscript. All authors contributed to the editing and development of the manuscript.

\section{Acknowledgments}

This work was supported by the National Science Foundation (CBET-1236029 and EAGER 00126963 to T.S.A.) and the National Institutes of Health (R01HL114907 to T.S.A). This paper is subject to the NIH Public Access Policy.

\section{Competing interests}

The authors declare no competing interests.

\section{References}

1. Tong, W., X. Cao, S. Wen, R. Guo, M. Shen, J. Wang, and X. Shi. 2012. Enhancing the specificity and efficiency of polymerase chain reaction using polyethyleneimine-based derivatives and hybrid nanocomposites. Int. J. Nanomedicine 7:1069-1078.

2. Bai, Y., Y. Cui, G.C. Paoli, C. Shi, D. Wang, and X. Shi. 2015. Nanoparticles affect PCR primarily via surface interactions with PCR components: using amino-modified silica-coated magnetic nanoparticles as a main model. ACS Appl. Mater. Interfaces 7:13142-13153.

3. Cui, D., F. Tian, Y. Kong, I. Titushikin, and H. Gao. 2004. Effects of single-walled carbon nanotubes on the polymerase chain reaction. Nanotechnology 15:154-157.

4. Zhang, Z., M. Wang, and H. An. 2007. An aqueous suspension of carbon nanopowder enhances the efficiency of a polymerase chain reaction. Nanotechnology 18:355706.

5. Zhang, Z., C. Shen, M. Wang, H. Han, and X. Cao. 2008. Aqueous suspension of carbon nanotubes enhances the specificity of long PCR. Biotechniques 44:537-542.

6. Li, H., J. Huang, J. Lv, H. An, X. Zhang, Z. Zhang, C. Fan, and J. Hu. 2005. Nanoparticle PCR: Nanogold-assisted PCR with enhanced specificity. Angew Chem Int Ed Engl. 44:5100-5103.

7. Nie, L., L. Gao, X. Yan, and T. Wang. 2007. Functionalized tetrapod-like $\mathrm{ZnO}$ nanostructures for plasmid DNA purification, polymerase chain reaction and delivery. Nanotechnology 18:015101.

8. Li, M., Y.-C. Lin, C.-C. Wu, and H.-S. Liu. 2005. Enhancing the efficiency of a PCR using gold nanoparticles. Nucleic Acids Res. 33:e184.

9. Cao, X., J. Chen, S. Wen, C. Peng, M. Shen, and X. Shi. 2011. Effect of surface charge of polyethyleneimine-modified multiwalled carbon nanotubes on the improvement of polymerase chain reaction. Nanoscale 3:1741-1747.

10. Wang, L., Y. Zhu, Y. Jiang, R. Qiao, S. Zhu, W. Chen, and C. Xu. 2009. Effects of Quantum Dots in Polymerase Chain Reaction. J. Phys. Chem. B 113:7637-7641.

11. Abdul Khaliq, R., R. Kafafy H.M. Salleh, and W.F. Faris. 2012. Enhancing the efficiency of polymerase chain reaction using graphene nanoflakes. Nanotechnology 23:455106.

12. Yi, C., C.-C. Fong, W. Chen, S. Qi, C.-H. Tzang, S.-T. Lee, and M. Yang. 2007. Interactions between carbon nanotubes and DNA polymerase and restriction endonucleases. Nanotechnology 18:025102.

13. Sang, F.M., Y. Sun, Z. Xu, Y.S. Wang, and Z.Z. Zhang. 2013. Investigation of the interference of carbon nanomaterials with SYBR Green I-based real-time PCR. Adv. Mat. Res. 785-786:550-555.

14. Wan, W., J.T. Yeow, and M.I. Van Dyke. 2009. Size-dependent PCR inhibitory effect induced by gold nanoparticles. Conf Proc IEEE Eng Med Biol Soc. 2009:2771-2774

15. Vu, B.V., D. Litvinov, and R.C. Willson. 2008. Gold nanoparticle effects in polymerase chain reaction: favoring of smaller products by polymerase adsorption. Anal. Chem. 80:5462-5467.

16. Morikawa, M., Y. Kuboki, and F. Watari. 2012. Evaluation of protein adsorption to carbon nanotubes having different property, and identification of adsorbed proteins. Nano Biomed. 4:66-75.

17. Morikawa, M., Y. Kuboki, T. Akasaka, S. Abe, and F. Watari. 2012. Effect of pretreatments to carbon nanotubes on protein adsorption. Nano Biomed. 4:35-41.

Received 17 February 2017; accepted 30 June 2017.

Address correspondence to Tara Sabo-Attwood, Department of Environmental and Global Health, University of Florida, Box 110885, 2187 Mowry Road, Gainesville, FL 32611. E-mail: sabo@phhp.ufl.edu

To purchase reprints of this article, contact: biotechniques@fosterprinting.com 